1 and 2 of ref. 2; and Table 2 of ref. 3). This distinct lack of tropism (or the more universal permissiveness of PBMCs for HIV-1 regardless of the specific envelope regions) was not discussed in these papers.

We feel that this result is important and deserves additional comment. It suggests that viral envelope elements that otherwise abolish infectivity of cell lines are tolerated to a significant degree in human primary PBMCs, thereby suggesting fundamental differences of viral entry at the cellular level for these cells. In support of this concept of 'universal tropism', 66 isolates were found to replicate primary PBMCs in a similar fashion regardless of the clinical stage of the disease $^{5}$. Furthermore, this fundamental difference, which we describe as "enhanced permissivity", appears also to be related to a more soluble CD4 (sCD4)resistant state. In recent experiments, when PBMCs instead of transformed human $\mathrm{T} 4$ cell lines were used in infectivity titration experiments designed to study the decay rate of HIV-1, a doubling of the infectivity half-life, from 36 to 53 hours, was observed ${ }^{6}$. The sensitivity to sCD4 blocking of viral infection was also reduced by about 20 -fold.

These cellular factors operating in conjunction with viral envelope factors could begin to explain the growing body of experimental evidence indicating the greater degree of resistance in SCD4 blocking and neutralization by antibodies observed for both primary isolates and laboratory strains. As we consider the issue of tropism and attempt to model more predictive in vitro systems of viral infectivity, an appreciation of both viral and cellular variables should improve our understanding and lead to more effective initial immunoprophylactic and therapeutic approaches. These results ${ }^{6}$, coupled to the more 'universal tropism' exhibited in the previous studies $^{1-3}$, imply that viral absorption, binding and penetration of HIV-1 is fundamentally a more efficient and permissive process in human PBMCs. The greater resistance to blocking and infection by SCD 4 and neutralizing antibodies, in addition to the prolonged infectivity exhibited during the decay studies, suggests that other cell surface factors are contributing to viral entry.

\section{Peter Nara}

Wen-Po Tsai

Hsiang-Fu Kung

Anton Minassian

Robert Garrity

National Cancer Institute,

Frederick,

Maryland 21702, USA

Jaap Goudsmit

\section{Gustaaf Rimmelzwaan}

Virology Department,

Academic Medical Centre,

Meibergdrees $151105 \mathrm{AZ}$.

Amsterdam,

The Netherlands

1. O'Brien, W. A. et al. Nature 348, 69-73 (1990)

2. Shioda, M., Levy. J. A. \& Cheng-Mayer. C. Nature 349 167-169 (1991).

3. Hwang, S. S.. Boyle, T. J., Lyerly, H. K. \& Cullen, B. R. Science 253, 71-74 (1991)

4. Nara, P. L., Garrity, R. \& Goudsmit, J. FASEB J. 5 2437-2455 (1991)

5. Lu, W. \& Andrien, J. J. Virol, 66, 334-340 (1992)

6. Layne. S. et al. Virology 189, 695-714 (1992).

\section{Even larger organisms}

SIR - Extremism, in the pursuit of science, is apparently no vice. We wish to contribute to the discussions about the largest living organisms ${ }^{1}$ which were stimulated by the nomination ${ }^{2}$ of Armillaria bulbosa as among the largest of living organisms. Two measures of size were reported: mass at $10,000 \mathrm{~kg}$ and area at 15 ha for this fungus. Two other rather more famous entities have traditionally headed the list of living behemoths: the giant sequoia at just less than 2,000,000 kg (ref. 3) and the blue whale at a paltry $180,000 \mathrm{~kg}$.

In the interests of big science, we wish to nominate the quaking aspen, Populus tremuloides, as the largest living organism whether measured by area or mass. At least one clone in the western United States cited in ref. 4 covered 43 ha, nearly three times the area reported for Armillaria. This aspen clone was estimated to contain 47,000 stems. An average individual aspen shoot (stem plus leaves and branches) would easily exceed $100 \mathrm{~kg}$ in mass while the accom- panying root system would probably exceed $30 \mathrm{~kg}$. Combination of these rather conservative figures produces an estimate of approximately $6,000,000 \mathrm{~kg}$ mass for that particular aspen clone, a number 60 times larger than the mass of the Armillaria thallus and more than triple that of the largest giant sequoia, the species most widely acknowledged as the mass champion of all plants ${ }^{2}$. Quaking aspen, the most widespread tree species in North America, can now take its rightful place as an acknowledged giant among giants.

Michael C. Grant

Jeffin B. Mitton

Yan. B. Linhart

Department of Environmental, Population and Organismic Biology,

University of Colorado,

Boulder, Colorado 80309, USA

1. Brasier, C. M. Nature 356, 382-383 (1992)

2. Smith, M. L., Bruhn, J. N. \& Anderson, J. B. Nature 356 428-431 (1992)

3. Elias, T. S. Field Guide to North American Trees (Grolier Book Clubs. Danbury, Connecticut, 1989)
4. Barnes, B. V. Forest Sci. 21, 319-328 (1975)

\section{Motor neuron disease model}

SIR - M. Sendtner et al. (Nature 358, $502-504 ; 1992)$ report that in a mouse mutant with progressive motor neuronopathy, ciliary neurotrophic factor (CNTF) released by implants of genetically modified fibroblasts increases the survival of facial motor neurons and prolongs the lifespan of the diseased animals. However, we became puzzled about the animal model that they used. In particular, the results in their Table 1 show a relatively modest loss of facial motor neurons at a time when the animals are terminally ill. Can such a small degree of motor neuron loss account for the death of these animals?

We turned to the original description of the mutant used $(\mathrm{H}$. Schmalbruch et al. J. Neuropath. exp. Neurol. 50, 192$204 ; 1991)$. To our surprise, the authors of this paper concluded that even at the terminal stage of the disease there was no loss of motor neurons. "The cervical and lumbar spinal cord of diseased mice in sections stained with cresyl violet appeared normal. It was not possible to identify unmarked sections from normal or diseased mice." In another part of the paper the authors say: "All nuclei of cranial nerves were present and did not reveal qualitative or obvious quantitative changes." This is in sharp contrast to the statement by Sendtner et al. that "The motor neurons of $\mathrm{pmn} / \mathrm{pmn}$ mice first undergo a reduction in cell size, then chromatolysis and finally cell death."

These discrepancies make us wonder whether Sendtner et al. used a different mutant, or whether the colony has changed. But if the original description of the disease is correct and the disease affects predominantly motor nerve fibres and not cell bodies as well as nerve fibres, then the interpretation of the results of Sendtner et al. is entirely different from that proposed by them, that is, that CNTF rescues motor neurons in this mouse mutant which, according to the previous description, suffers from no loss of motor neurons.

The results are no less interesting if genetically manipulated cell implants that release CNTF protect nerve fibres from dying back rather than rescuing motor neurons. However, the theoretical and clinical implications are entirely different.

Gerta Vrbová

Linda Greensmith

Katarzyna Sieradzan

Department of Anatomy and

Developmental Biology,

University College,

Gower Street.

London WC1E 6BT, UK 